\title{
An assessment of the histological criteria used to diagnose infection in hip revision arthroplasty tissues
}

R Pandey, E Drakoulakis, N A Athanasou

\begin{abstract}
Aim-To characterise the number and nature of the inflammatory cells seen in cases of septic or aseptic loosening of hip arthroplasty, and to establish reliable histological criteria to distinguish between these two conditions.

Methods-Histological examination of paraffin sections of periprosthetic tissues (pseudocapsule, femoral and acetabular pseudomembranes) of 523 cases of aseptic loosening and 79 cases of microbiology culture proven septic loosening. The cellular composition of the inflammatory cell infiltrate was determined semiquantitatively.

Results-The finding of a $2+$ or greater neutrophil polymorph infiltrate (one or more cells per high power field $(\times 400)$ on average after examination of 10 fields) in arthroplasty tissues correlated strongly with the microbiological diagnosis of septic loosening: diagnostic sensitivity $100 \%$, specificity $97 \%$, accuracy $99 \%$, positive predictive value $92 \%$, negative predictive value $100 \%$. The finding of a $3+$ neutrophil polymorph infiltrate (five or more cells on average per high power field) had a diagnostic sensitivity of $72 \%$, specificity $100 \%$, accuracy $98 \%$, positive predictive value $100 \%$, and negative predictive value $\mathbf{9 7 \%}$. In some cases of septic loosening the finding of a heavy lymphocytic and plasma cell infiltrate was of low diagnostic sensitivity. A neutrophil polymorph infiltrate (generally less than one cell per 10 high power fields) was also seen in cases of aseptic loosening.
\end{abstract}

Conclusions-The presence of $2+$ or more (more than one neutrophil polymorph per high power field $(\times 400)$ on average after examination of at least 10 high power fields) in periprosthetic tissues provides the most sensitive and accurate histopathological criterion for distinguishing between septic and aseptic loosening of hip arthroplasty.

(f Clin Pathol 1999;52:118-123)

Keywords: arthroplasty; infection; histological diagnosis

artment of Pathology, Nuffield Orthopaedic Centre N A Athanasou

Correspondence to: Dr Athanasou.

Accepted for publication 10 November 1998 ment and outcome of these two conditions differ markedly. ${ }^{1-4} \mathrm{~A}$ diagnosis of septic loosening has significant clinical and cost implications in terms of the need for prolonged antibiotic treatment, longer hospital stay, and planning for a second surgical procedure. Failure to establish a diagnosis of infection as a cause of implant loosening has equally serious consequences: persistence of the infection leads to early failure of a revision arthroplasty with continuing loss of bone around a prosthesis and, in many cases, the need for a second often more difficult and extensive surgical procedure.

Clinical and laboratory features indicative of acute infection may be present in some (but not all) cases of early arthroplasty failure caused by sepsis. ${ }^{56}$ However, in delayed or late onset septic loosening, clinical features of infection are often absent and radiological and laboratory investigations - such as gallium scans, ${ }^{7}$ erythrocyte sedimentation rate (ESR), ${ }^{8}$ $\mathrm{C}$ reactive protein, ${ }^{9}$ and hip aspiration before surgery $^{7}{ }^{10}{ }^{11}$-are not sensitive or specific enough to distinguish reliably between septic and aseptic loosening.

Several studies have shown that histological examination of arthroplasty tissues is useful in establishing a diagnosis of septic loosening. ${ }^{12-15}$ This is being increasingly employed intraoperatively, the results of frozen section histology providing a guide as to whether a one or two stage arthroplasty procedure needs to be carried out. ${ }^{13-15}$ The histological feature that suggests the pathological diagnosis of septic loosening is the presence of numerous inflammatory cells, particularly neutrophil polymorphonuclear leucocytes (neutrophils) in the arthroplasty pseudomembrane or pseudocapsule. Although the reliability of this investigation in diagnosing infection has been questioned, ${ }^{1617}$ all observers agree that the presence of numerous neutrophils in periprosthetic tissues correlates strongly with septic loosening. However, it is not clear how extensive the neutrophil infiltrate needs to be before it can be regarded as diagnostic of infection, ${ }^{12-15}$ while the significance of other inflammatory cells, such as lymphocytes and plasma cells, in distinguishing between septic and aseptic loosening is also uncertain. ${ }^{12}{ }^{13}$

In this study we have analysed the nature of inflammatory cells (other than wear particle associated mononuclear phagocytes) in periprosthetic tissues taken from a large number of cases of arthroplasty failure in which a definite clinical and microbiological diagnosis of septic or aseptic loosening was established. Both clinical and microbiological evidence of infection or the absence of infection was sought 
to establish the diagnosis of septic and aseptic loosening respectively, as it has been shown previously that failure to culture organisms from orthopaedic specimens does not exclude the presence of infection. ${ }^{18}$ Thus the aim of this study was to establish precise histological criteria whereby septic and aseptic loosening can be reliably distinguished.

\section{Methods}

CASES EXAMINED

Periprosthetic tissues of 602 cases of hip revision arthroplasty, carried out between 1992 and 1996, were submitted for histological examination. In all cases, the original hip arthroplasty was carried out for osteoarthritis. The cases examined were divided into two groups.

Group 1, septic loosening-79 cases of septic loosening where an organism had been isolated on bacteriological culture (see below); in all cases antibiotic treatment was given postoperatively to treat the infection. Clinical and investigative findings which strongly suggested a diagnosis of septic loosening included two or more of the following features ${ }^{145}$ : early failure of the prosthesis; constant pain or persistent poor function of the prosthesis; a history of wound infection or sinus formation following primary arthroplasty; raised $\mathrm{C}$ reactive protein and/or erythrocyte sedimentation rate; bone scintigraphy or other radiological evidence suggestive of infection. As the aim of this study was to provide benchmark figures whereby cases of septic and aseptic loosening could be distinguished histologically, cases in which there were equivocal microbiological findings (that is, not considered significant clinically or microbiologically) were not included for assessment in this study.

Group 2, aseptic loosening - 523 cases of aseptic loosening in which there were no clinical or laboratory features to suggest that loosening of the prosthesis was caused by infection and where an organism was not isolated by microbiological culture of periprosthetic tissues.

\section{HISTOLOGICAL METHODS}

Specimens from the joint pseudocapsule and from the femoral and acetabular pseudomembrane were submitted for histology; these were routinely sent before preoperative antibiotics were given. Specimens were fixed in formalin before sampling and then routinely processed; $5 \mu \mathrm{m}$ paraffin sections were cut and stained with haematoxylin and eosin (H\&E); Gram staining was carried out in all cases diagnosed as septic loosening.

We noted the presence of inflammatory cells in the periprosthetic tissues (other than wear particle associated macrophages and macrophage polykaryons). Inflammatory changes in infected arthroplasty tissues were often found to be quite focal rather than diffusely distributed. In some cases, marked differences in the composition of the inflammatory cell infiltrate were seen in specimens from the different periprosthetic tissues sampled, or even from different samples taken from a single periprosthetic tissue specimen. Even within a histologi- cal section of a sampled tissue there was also some variation in the extent of the inflammatory infiltrate. We thus first scanned sections of tissue from each specimen to find the most florid areas of inflammatory cell infiltration; then, using a modification of the histological scoring method of Mirra et $a l,{ }^{12}$ we calculated the extent of the acute and chronic inflammatory cell infiltrate semiquantitatively. We examined at least 10 high power fields $(\times 400 ; 3.15$ $\mathrm{mm}^{2}$ ) in five different areas of each histological section and calculated the average score of the five areas for infiltration by neutrophils, eosinophil polymorphs, lymphocytes, and plasma cells. The scores were categorised as follows: $0=$ absent; $1+=$ fewer than 1 cell per 10 high power fields $(\times 400) ; 2+=1-5$ cells per high power field (average); $3+=>5$ cells per high power field (average).

\section{MICROBIOLOGICAL METHODS}

Microbiological processing of specimens was as previously described. ${ }^{13}{ }^{16}$ Briefly, at least two independent specimens of the tissue joint capsule and arthroplasty membrane were submitted for microscopy and culture from each case. Specimens were transferred to universal receptacles containing $3 \mathrm{ml}$ of peptone broth and Ballotini glass balls. The tissue was dispersed as far as possible by shaking. Aliquots of the processed tissue in peptone were plated onto two blood agar plates ( $5 \%$ horse blood)-one aerobic and one anaerobic - and onto a chocolate agar plate. The remainder of the tissue was transferred to tryptone soy broth, $0.1 \%$ agar, for enrichment culture. ${ }^{13}$ The aerobic blood agar plates were incubated in $10 \% \mathrm{CO}_{2}$, and the anaerobic plate in an anaerobic chamber. The aerobic blood agar plate and chocolate plate were incubated for 48 hours. The anaerobic plates and enrichment broths were incubated for five days. Organisms were identified using routine laboratory methods.

Isolates were considered significant if they grew on direct plating, or if an indistinguishable strain grew on enrichment in more than one culture, particularly from three or more independent specimens. ${ }^{16}{ }^{19} 20$ Organisms were considered contaminants if distinguishable strains were isolated from different enrichment broths, and no growth occurred on direct plating. A single isolate of a strain from only one enrichment culture was considered not significant. Antibiotic treatment was individualised on the basis of the culture and antibiotic sensitivity results; the most commonly employed antibiotics in the treatment of septic loosening were penicillins, cephalosporins, and vancomycin.

\section{STATISTICAL METHODS}

For establishing a histological diagnosis of infection, we calculated the sensitivity, specificity, accuracy, and the positive and negative predictive values (compared with the results of bacteriological culture) of the presence of $3+$ and $[>2+]$ infiltration by neutrophils, lymphocytes, and plasma cells. ${ }^{21}$ 
Table 1 Details of inflammatory cell infiltration in septic and aseptic loosening of hip arthroplasty

\begin{tabular}{|c|c|c|}
\hline Inflammatory cell & $\begin{array}{l}\text { Number } \\
\text { of cases }\end{array}$ & $\begin{array}{l}\text { Degree of } \\
\text { infiltration } \\
\text { (No of affected } \\
\text { cases) }\end{array}$ \\
\hline \multicolumn{3}{|c|}{ Group 1: septic loosening (79 cases) } \\
\hline Neutrophil polymorphs & 79 & $\begin{array}{l}1+(0) \\
2+(13) \\
3+(66)\end{array}$ \\
\hline Lymphocytes & 79 & $\begin{array}{l}1+(18) \\
2+(42) \\
3+(19)\end{array}$ \\
\hline Plasma cells & 44 & $\begin{array}{l}1+(17) \\
2+(15) \\
3+(12)\end{array}$ \\
\hline Eosinophil polymorphs & 4 & $\begin{array}{l}1+(2) \\
2+(2) \\
3+(0)\end{array}$ \\
\hline \multicolumn{3}{|c|}{ Group 2: aseptic loosening (523 cases) } \\
\hline Neutrophil polymorphs & 63 & $\begin{array}{l}1+(56) \\
2+(7) \\
3+(0)\end{array}$ \\
\hline Lymphocytes & 409 & $\begin{array}{l}1+(258) \\
2+(127) \\
3+(24)\end{array}$ \\
\hline Plasma cells & 38 & $\begin{array}{l}1+(25) \\
2+(9) \\
3+(4)\end{array}$ \\
\hline Eosinophil polymorphs & 18 & $\begin{array}{l}1+(5) \\
2+(11) \\
3+(2)\end{array}$ \\
\hline
\end{tabular}

Sensitivity was calculated as the proportion of infected arthroplasties (that is, microbiological culture proven) correctly identified by histological examination (the number of true positive results divided by the sum of true positive and false negative results).

Specificity was calculated as the proportion of non-infected hips (that is, culture negative) correctly identified (true negative results divided by the sum of true negative and false positive results).

Accuracy was calculated as the ratio of true positive and true negative results divided by the total number of results.

The positive predictive value was calculated as the ratio of true results to the total number confirmed as infected by bacteriology.

The negative predictive value was calculated as the ratio of true negative results to the number of cases found not to be infected.

\section{Results}

A summary of the histological features noted in group 1 cases of septic loosening and group 2 cases of aseptic loosening diagnosed by microbiological culture is shown in table 1 . There was no significant difference in sex, age, or laterality between the two groups. The mean time since installation of the prosthesis was 3.6 years in cases of septic loosening and 7.2 years for aseptic loosening. Table 2 shows the sensitivity, specificity, accuracy, and positive and negative predictive values of the presence of a $3+$ and $[>2+]$ infiltrate of neutrophils, lymphocytes, and plasma cells.

GROUP 1: SEPTIC LOOSENING

All 79 cases of culture proven septic loosening showed evidence of an acute and chronic inflammatory infiltrate in one or more periprosthetic tissues. Neutrophils were the most common inflammatory cell seen in the
Table 2 Assessment of the inflammatory cell infiltrate in infected hip revision arthroplasty tissues

\begin{tabular}{|c|c|c|c|c|c|c|}
\hline & \multicolumn{2}{|c|}{$\begin{array}{l}\text { Neutrophil } \\
\text { polymorphs }\end{array}$} & \multicolumn{2}{|c|}{ Plasma cells } & \multicolumn{2}{|c|}{ Lymphocytes } \\
\hline & $>2+$ & $3+$ & $>2+$ & $3+$ & $>2+$ & $3+$ \\
\hline Sensitivity & $100 \%$ & $72 \%$ & $21 \%$ & $8 \%$ & $63 \%$ & $14 \%$ \\
\hline Specificity & $97 \%$ & $100 \%$ & $95 \%$ & $98 \%$ & $55 \%$ & $91 \%$ \\
\hline Accuracy & $99 \%$ & $98 \%$ & $89 \%$ & $88 \%$ & $72 \%$ & $86 \%$ \\
\hline PPV & $92 \%$ & $100 \%$ & $62 \%$ & $75 \%$ & $25 \%$ & $44 \%$ \\
\hline NPV & $100 \%$ & $97 \%$ & $89 \%$ & $88 \%$ & $91 \%$ & $89 \%$ \\
\hline
\end{tabular}

NPV, negative predictive value; PPV, positive predictive value.

pseudomembrane or pseudocapsule of infected arthroplasty specimens. This neutrophil infiltrate was generally heavy, most commonly grade $3+$ (that is, more than five cells per high power field on average), but in over $16 \%$ of infected cases the neutrophil infiltrate was $2+$ (1-5 cells per high power field on average). The finding of a $3+$ neutrophil infiltrate had a diagnostic sensitivity of $72 \%$, a specificity of $100 \%$, accuracy of $98 \%$, a positive predictive value of $100 \%$, and a negative predictive value of $97 \%$. The finding of $[>2+]$ neutrophil infiltrate had a diagnostic sensitivity of $100 \%$, a specificity $97 \%$, accuracy $99 \%$, a positive predictive value of $92 \%$, and a negative predictive value of $100 \%$. Organisms were identified by Gram staining in only 17 cases of septic loosening (that is, approximately $21.5 \%$ of cases).

A heavy acute inflammatory infiltrate was almost always accompanied by the presence of other inflammatory cells. This was usually in the form of a prominent lymphocytic infiltrate, most commonly $2+$ but sometimes $3+$ in degree. As a prominent lymphocytic infiltrate was not uncommonly also found in cases of aseptic loosening (see below), the presence of numerous lymphocytes in periprosthetic tissues was not a sensitive marker of septic loosening; diagnostic specificity and accuracy were also much less than for neutrophils-91\% and $86 \%$ respectively for a $3+$ lymphocytic infiltrate. Plasma cells were seen in over $50 \%$ of cases of septic loosening and were numerous $(2+)$ or very numerous (3+) in 27 of 44 cases where these cells were noted. Although, like lymphocytes, a $2+$ or more plasma cell infiltrate was not a sensitive marker of septic loosening, it was more commonly found in infected than in uninfected hip revision arthroplasty tissues, showing relatively high diagnostic specificity and negative predictive value in cases of septic loosening. Lymphocytes and plasma cells were seen together in large lymphoid aggregates in seven cases of septic loosening; one of these cases also showed the presence of large lymphoid follicles having a germinal centre within the arthroplasty pseudomembrane and pseudocapsule.

Although the pattern of inflammatory cell infiltration within the arthroplasty pseudomembrane and pseudocapsule was often variable, we noted that in all cases of septic loosening more than one specimen always showed evidence of a $2+$ neutrophil infiltrate. In most cases of septic loosening there was a diffuse $2+$ or $3+$ neutrophil infiltrate among which were scattered other inflammatory cells. In some cases focal collections of $2+$ or $3+$ neutrophils 
were found within periprosthetic tissues, usually just beneath the surface of the pseudocapsule or pseudomembrane, which was commonly ulcerated. More than two such focal collections of $2+$ or $3+$ neutrophil infiltrate were noted in all cases of septic loosening. In a few cases, a heavy diffuse lymphocytic and plasma cell infiltrate predominated and masked the presence of neutrophils, but in these cases focal areas of $2+$ or $3+$ neutrophil infiltration could be found on careful searching.

GROUP 2: ASEPTIC LOOSENING

Histological examination of tissues taken from cases diagnosed as aseptic loosening (on the basis of the absence of clinical or laboratory features suggestive of active infection and the failure to isolate or culture an organism) showed a variable but generally heavy macrophage and macrophage polykaryon response to wear particles within connective tissue of the arthroplasty pseudomembrane and pseudocapsule. Scattered bone fragments, haemosiderin, focal areas of degenerate collagen, fibrocartilaginous metaplasia, and areas of reparative fibrous and granulation tissue were also observed in some specimens.

Inflammatory cells other than wear particle associated macrophages and macrophage polykaryons were also commonly present in the periprosthetic tissues of cases of aseptic loosening. Lymphocytes were most frequently noted. They were found in just over two thirds of all specimens of aseptic loosening, most commonly as a minor $(1+)$ inflammatory component $(49 \%)$. A $2+$ and $3+$ lymphocytic infiltrate was noted in approximately $24 \%$ and $5 \%$ of cases, respectively. Not uncommonly, lymphocytes were found aggregated around small blood vessels within the deep tissue of the pseudocapsule and pseudomembrane. Aggregates of lymphocytes were also commonly seen around large fragments of PMMA and, in general, a heavy lymphocytic infiltrate was seen in failed arthroplasty membranes which contained heavy deposits of polymeric or metallic wear particles.

Plasma cells were not commonly seen in uninfected arthroplasty tissues, being found in only $7 \%$ of cases. Where identified, they were present in small $(1+)$ numbers, usually in the context of heavy wear particle (particularly metallic wear particle) deposition. In all cases where a plasma cell infiltrate was noted there was also a prominent $2+$ or $3+$ lymphocytic infiltrate. Plasma cells were sometimes scattered among lymphocytes in small lymphoid aggregates. In two cases, where there was a heavy (3+) lymphocyte and plasma cell infiltrate, a very prominent $(3+)$ eosinophil polymorph infiltrate was also noted within the arthroplasty pseudomembrane. In these two cases, there was abundant polymeric and metallic wear particle deposition in the pseudocapsule and pseudomembrane. In the other 16 cases where eosinophil polymorphs were identified in the tissues, lymphocytes were also noted and, in six of these cases, plasma cells were present.
Isolated or occasional $(1+)$ neutrophils were seen in approximately $10 \%$ of periprosthetic tissues derived from cases of aseptic loosening. A $2+$ polymorph infiltrate was noted in seven cases (that is, approximately $1 \%$ of cases of aseptic loosening); this was most commonly seen as discrete focal collections of neutrophils among other inflammatory cells in the sublining tissue of the pseudocapsule (four cases) and the acetabular pseudomembrane (two cases); it was also seen in one case in the femoral pseudomembrane. Such foci of $2+$ neutrophil infiltration were always found in the superficial zone of the pseudocapsule or pseudomembrane, often near an area of ulceration. With the exception of two cases, which showed $2+$ lymphocytes and $1+$ plasma cells in the inflammatory infiltrate, the periprosthetic tissues of cases of aseptic loosening containing $2+$ neutrophils showed only a macrophage and giant cell response to wear particles. Organisms were not identified by Gram staining in culture negative specimens where this investigation was carried out.

\section{Discussion}

The presence of acute and chronic inflammatory cells in periprosthetic tissues has been shown to correlate closely with the diagnosis of septic loosening, but there is controversy over the nature and extent of the inflammatory cell infiltrate needed to establish a diagnosis of septic loosening. Mirra et al found a heavy inflammatory cell infiltrate on frozen section histology in all 15 cases which showed clinical or bacteriological evidence of infection, and an absence of neutrophil polymorphs in 21 uninfected cases. ${ }^{12}$ Feldman et $a l,{ }^{14}$ using similar histological criteria (that is, five neutrophil polymorphs per high power field), noted similar changes in 10 bacteriologically proven cases. In a series of 85 cases of hip arthroplasty, ${ }^{22}$ Pizzoferrato et al obtained 10 false negative diagnoses but confirmed the value of the histological criteria established by Mirra et al. Fehring and McAlister, ${ }^{17}$ in a series of 107 joint revisions, used the criteria of Mirra et $a l$ and concluded that frozen section histology was not sufficiently sensitive to detect occult septic loosening. In a previous frozen section histology study of 106 revision hips and knees, we found that an average of one or more neutrophils or plasma cells per high power field correlated closely with a microbiological diagnosis of infection. ${ }^{13}$ Lonner et al, ${ }^{15}$ studying 175 hip and knee arthroplasties, found that at least 10 neutrophils per high power field were required to establish a diagnosis of infection, and that five to nine neutrophils per high power field were not necessarily consistent with infection. Demonstrating the presence of neutrophils in aspiration biopsies of the joint pseudocapsule has also been shown to be useful in the preoperative diagnosis of septic loosening. ${ }^{9} 10$

Our results suggest that the presence of a significant number of neutrophils is the most important histological feature in establishing a diagnosis of septic loosening. Although in most cases $(84 \%)$, this neutrophil polymorph 
infiltrate is heavy $(3+$, more than five cells per high power field on average), we found that it was less pronounced $(2+$, one or more cells per high power field on average) in a significant number of cases. The relatively low sensitivity $(72 \%)$ and positive predictive value $(84 \%)$ obtained by using more than five neutrophils per high power field as the cut off point for the histological diagnosis of septic loosening suggests that that figure is too high. In our series such a course would have resulted in failure to confirm the microbiological diagnosis in $16 \%$ of the cases. However, by making the cut off point one neutrophil per high power field (on average), the diagnostic sensitivity, accuracy, and positive predictive value of histology is markedly enhanced. The finding of isolated (1+) neutrophil polymorphs in periprosthetic tissues (that is, less than one neutrophil per 10 high power fields) was not found to correlate with septic loosening and was seen in almost $11 \%$ of cases of aseptic loosening. We found that Gram staining was positive in only $21.5 \%$ of the cases of septic loosening. This confirms the findings of previous studies which concluded that positive identification of organisms should not be regarded as necessary for the histological diagnosis of septic loosening. ${ }^{13} 23$

Apart from wear particle associated macrophages and macrophage polykaryons, the presence of lymphocytes or plasma cells was not of itself found to be useful as a predictive marker of septic loosening. A $1+$ or $2+$ infiltrate of lymphocytes was not uncommonly found in the periprosthetic tissues of cases of aseptic loosening. However, the finding of a heavy (3+) lymphocytic infiltrate in periprosthetic tissues-although not in itself a sensitive marker of septic loosening-showed sufficient specificity for septic loosening to serve as a marker of the need for a careful search of periprosthetic tissues for areas of significant neutrophil infiltration. The finding of a $2+$ or $3+$ plasma cell infiltrate also showed low diagnostic sensitivity and positive predictive value but-particularly in the case of $3+$ plasma cells-was found to be a relatively specific marker for septic loosening. Other inflammatory cells, such as eosinophil polymorphs, were not commonly seen in periprosthetic tissues derived from cases of septic or aseptic loosening.

Although inflamed granulation tissue and an inflammatory exudate containing numerous neutrophils were the most common histological findings in infected cases, the severity and distribution of these inflammatory changes not only varied from case to case but also between different specimens taken from the same case. Thus a feature highlighted by this study was the importance of adequately sampling all periprosthetic tissues which are submitted for histology. In some cases, the neutrophil infiltrate was not diffuse but was focally distributed and it was necessary for several samples to be taken before the acute inflammatory changes diagnostic of infection could be found. The failure to obtain tissue from an inflammatory focus within the joint capsule could explain the low sensitivity of histology in the diagnosis of infection in some biopsy series. ${ }^{91622}$

In the course of this and our previous study ${ }^{13}$ we also found it useful to make a note of the clinical features that either suggested the diagnosis of septic loosening or helped to explain the histological findings in such cases. These included short duration of a prosthesis in situ and evidence of wound infection after primary arthroplasty. We also noted that a fracture occurring around an aseptically loose prosthesis could result in histological changes that could be confused with septic loosening. Haemorrhage on the pseudomembrane surface or within arthroplasty tissues results in the focal appearance of red cells and occasionally large numbers of polymorphs in this location; this histological feature in aseptic loosening needs to be distinguished from an inflammatory fibrinous exudate accompanying infection. Another cause of possible confusion is the reparative cellular fibrous and granulation tissue that is often found in periprosthetic tissues obtained from cases of aseptic loosening; congested small vessels in the tissue may contain neutrophils, which should not be counted when analysing the inflammatory infiltrate. We recommend that in distinguishing between septic and aseptic loosening, histological assessment should be confined to the number and nature of inflammatory cells present within extravascular periprosthetic tissue rather than on the surface. ${ }^{13}$

It should also be noted that the findings in this study relate only to cases in which the primary arthroplasty was originally carried out for degenerative joint disease and not for rheumatoid arthritis or other forms of inflammatory arthropathy, in which a heavy chronic inflammatory infiltrate is commonly found within periprosthetic tissues.

\section{CONCLUSIONS}

Our results strongly suggest that histological findings in periprosthetic tissues are useful in distinguishing cases of septic from aseptic loosening. On the basis of our results we would recommend that the finding of $3+$ neutrophils (more than five per high power field on average) should be regarded as diagnostic of infection and that $2+$ neutrophils (1-5 cells per high power field on average) should be considered highly suggestive of infection. The presence of other inflammatory cell elements, particularly a $2+$ or $3+$ plasma cell infiltrate or a $3+$ lymphocytic infiltrate within the periprosthetic tissues, although not diagnostic of infection, should be noted and a careful search of the tissues undertaken for the presence of neutrophil polymorphs. These findings should be useful in confirming a clinical diagnosis of septic loosening on routine histology of periprosthetic tissues, particularly in those cases where the microbiological findings are negative or equivocal. They also provide a guide to the criteria for distinguishing between septic and aseptic loosening on frozen section histology. Although the frozen section diagnosis of septic loosening is based on similar criteria, the morphological identification of neutrophils and 
their distinction from other inflammatory elements within periprosthetic tissues is not as straightforward in frozen sections as in paraffin sections; sampling error is also more of a problem as there is usually less tissue to examine.

We would like to thank Mrs M Pearce and Charlotte Young for typing the manuscript. NAA is a member of the OSIRIS study group.

1 Antti-Poika I, Josefsson G, Konttinen Y, et al. Hip 1990;61:163-9.

2 Morschen E, Babst R, Jenny H. Treatment of infected joint arthroplasty. Int Orthop 1990;14:161-5.

3 Walenkamp GH. Exchange arthroplasty for infected hip and knee prostheses. In: Coombs R, Fitzgerald RH, eds. Infection in the orthopaedic patient. London: Butterworths, Infection in the

4 Masterson EL, Basson AM, Duncan CP. Treatment of infection at the site of total hip replacement. F Bone foin Surg Br 1997;79B:1740-8.

5 Rothman RH, Hozack WJ. Complications of total hip arthroplasty. Philadelphia: WB Saunders, 1988.

6 Hamblen DL. Diagnosis of infection and the role of permanent excision arthroplasty. Orthop Clin North Am 1993;24: 743-9.

7 Kraemer WJ, Saplys R, Waddell JP, et al. Bone scan, gallium scan and hip aspiration in the diagnosis of infected total hip arthroplasty. F Arthroplasty 1993;8:611-15.

8 Carlsson AS. Erythrocyte sedimentation rate in infected and non infected total hip arthroplasties. Acta Orthop Scand 1978;49:287-90.

9 Sanzen L, Carlsson AS. The diagnostic value of CRP in infected total hip arthroplasty. I Bone foint Surg $\mathrm{Br}$ infected total hip

10 Gould ES, Pollen HG, Boben SE. Role of routine percutaneous hip aspirations prior to prosthesis revision. Skel neous hip aspirations pric
Radiol 1990;19:427-50.
11 Barrack RL, Harris WH. The value of aspiration of the hip joint before total hip arthroplasty. I Bone foint Surg Am 1993;75A:66-76.

12 Mirra JM, Amstutz HC, Matos M, et al. The pathology of the joint tissues and its clinical relevance in prosthesis failure. Clin Orthop 1976;117:221-40.

13 Athanasou NA, Pandey R, deSteiger R, et al. Diagnosis of infection by frozen section during revision arthroplasty. $\mathcal{F}$ Bone foint Surg Br 1995;77B:28-33.

14 Feldman DS, Lonner JH, Desai P, et al. The role of intraoperative frozen sections in revision total joint arthroplasty. $\mathcal{F}$ Bone foint Surg Am 1995;77A:1807-913.

15 Lonner JH, Desai P, Dicesare PE, et al. The reliability of analysis of intraoperative frozen sections for identifying active infection during revision hip or knee arthroplasty. $\mathcal{F}$ Bone foint Surg Am 1996;78A:1553-8.

16 Brause BD. Infected orthopaedic prosthesis. In: Bisno AL, Waldvogel FA, eds. Infections associated with indwelling medical devices. Washington DC: American Society for Microbiology, 1989:111-27.

17 Fehring TK, McAlister JA. Frozen histologic section as a guide to sepsis in revision joint arthroplasty. Clin Orthop 1994;304:229-32.

18 Perry CR, Pearson RL, Miller GA. Accuracy of cultures of material from swabbing of the superficial aspect of the wound and needle biopsy in the preoperative assessment of osteomyelitis. F Bone foint Surg Am 1991;73A:745-9.

19 Balows A, Hausler WJ. Manual of clinical microbiology. Washington DC: American Society for Microbiology, 1991.

20 Atkins B, Athanasou NA, Deeks JJ, et al. Prospective evaluation of criteria for the microbiological diagnosis of prosthetic joint infection at revision arthroplasty. F Clin Microbiol 1998;36:2932-9.

21 Armitage P. Statistical methods in medical research. Oxford: Blackwell, 1971

22 Pizzoferrato A, Fiori F, Savarino L. Microbiological investigation on 161 cases of hip endo-arthroprosthesis failure. Chir Organi Mov 1981;66:297-307.

23 Chimento GF, Finger S, Barrack RL Gram stain detection of infection during revision arthroplasty. $\mathcal{F}$ Bone foint Surg Br 1996;78B:838-9. 